\section{In Memory of Raffaele Gianotti}

Prof. Raffaele Gianotti ("Raf" for friends) was born on July 7th,1959 in Milan (Italy). His father, Prof. Ferdinando Gianotti (1920-1984), was a famous pediatric dermatologist that gave his name to the "papular acrodermatitis of childhood" (Gianotti-Crosti Syndrome).

Raffaele graduated cum laude in Medicine in 1985 at the University of Milan. In 1988 he completed the residency in Dermatology and in 1992 he got also his second specialization in Surgical Pathology. Soon after in 1993, he made a fellowship in Dermatopathology by Bernie Ackerman in New York. Back again in Milan, he started the work of his life of in Dermatopathology with a full researcher position at Istituto di Scienze Dermatologiche Fondazione Ospedale Maggiore Policlinico, Mangiagalli e Regina Elena. Between 20002005 he collaborated with H. Kutzner in Friedricshafen (Germany) on Lampyris101, an automatized software for the diagnosis of inflammatory skin diseases. In 2005, he also got the International Board Certification in Dermatopathology.
As academic, he published over 135 scientific papers on various topics on inflammatory and neoplastic skin diseases and reached an H-index of 27 (source: Scopus, last visited April 5, 2021). Particularly, he was recently very active in describing the histological features of COVID-19 infection in the skin and became very popular reporting the first proved Italian patient affected by COVID-19. ${ }^{1-4}$

Raf was also author of Dermosprint (https://www.dermosprint.com/), a freely available Dermatopathology collection with contributions by worldwide best dermatopathologists.

He passed away on March 27, 2021 at the age of 61 .

In his memory, we decided to publish posthumous in Dermatology Reports an editorial that Raf wrote for Dermosprint.

Ciao Raf! Rest in peace.

Cesare Massone, ${ }^{1}$ Antonio Perasole ${ }^{2}$ ${ }^{1}$ Dermatology Unit, Galliera Hospital, Genoa (Italy)

${ }^{2}$ Consultant Pathologist at Lifebrain s.r.l. Limena, Padua (Italy)

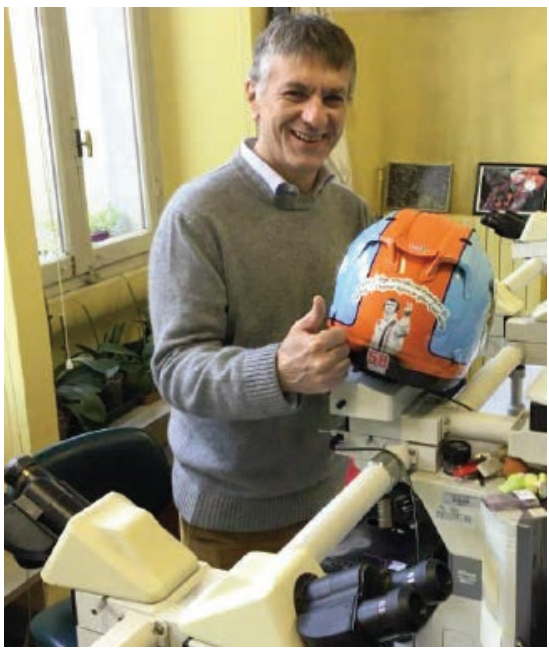

Raf Gianotti with both his work \& hobby: dermatopathology and motorbike.

\section{References}

1. Crosti A, Gianotti F. Dermatose éruptive acro-située d'origine probablement virosique [Eruptive dermatosis of probable viral origin situated on the acra]. Dermatologica 1957;115:671-7.

2. Gianotti R. SARS-CoV-2 and the skin, a hidden treasure. Dermatol Rep 2020;12: 8881.

3. Gianotti R, Barberis M, Fellegara G, et al. COVID-19-related dermatosis in November 2019: could this case be Italy's patient zero? Br J Dermatol 2021 Jan 7. doi: 10.1111/bjd.19804.

4. Gianotti R, Recalcati S, Fantini F, et al. Histopathological Study of a Broad Spectrum of Skin Dermatoses in Patients Affected or Highly Suspected of Infection by COVID-19 in the Northern Part of Italy: Analysis of the Many Faces of the Viral-Induced Skin Diseases in Previous and New Reported Cases. Am J Dermatopathol 2020;42: 564-70. 


\section{And... who is the best of all times in diagnosing melanocytic lesions?}

\section{Raffaele Gianotti}

Clinica Dermatologica, Università degli Studi di Milano, Italy

*published posthumous

A question came to my mind as I was writing about Ackerman and sharing all the thoughts of legends like LeBoit, Kutzner, and Weedon: "Of all times, who has been and who is the best dermatopathologist in the field of melanocytic lesions?" I am not talking about charisma, the number of index publications, awards received or the lectures held around the world. What I mean is who, sitting at the microscope, never misses a diagnosis, or if you prefer, makes the least wrong calls. How often have sports reporters asked themselves: "Who was the best tennis player of all time? And ... the football player? And ... the F.1 driver?" If nowadays Dante Alighieri wrote the Divina Commedia, written in a language typical of the 1300s, you could barely find it on the shelves of a supermarket. It is impossible to answer who's best when comparing individuals from different times.

Dear reader, be honest and admit to yourself that at least once in your life, you couldn't help thinking you were better at diagnosing than your colleague that works in a lab or a department hundreds of kilometers away from your workplace, whom you come across at a surgical pathology congress or inter-regional gatherings.

It is hard to determine who nowadays is the most gifted in examining a histological section on the controversial issue of Spitz nevus and spitzoid melanoma. What if I told you that my friend and colleague Giuseppe a fellow pathologist at a peripheral hospital of suburban town has an extremely high score in melanocytic lesions? Would you believe it? It could be true, but it is impossible to prove! In our job, there are no "scores", no timer running to measure up against, and no championship that rewards the best athlete of the year based on actual results.

Several years ago, 26736 melanomas were examined in an Australian study. It was established that only $4 \%$ of the patients with a Breslow index above $0.75 \mathrm{~mm}$ would not survive 20 years since the disease was first determined. I always asked myself: "How many of these four patients, before passing to a better place, manage to contact the pathologist who, perhaps ten years earlier, made a diagnosis of Spitz lesion?". In light of this study, you could decide to diagnose all of the histologic sections for pigmented lesions, even in presence of melanoma, as melanocytic nevus, as long as they don't go beyond the fateful $0.75 \mathrm{~mm}$. Probably no more than a single patient, or none at all, would contact the dermatopathologist who made the diagnose maybe it would be just one patient out of a whole pathologist carrier. And ... how about the patients who were mistakenly diagnosed with melanoma. No further news from them, whose only trouble will be to have frequent nightmares of the "sword of Damocles" in the shape of a melanoma hanging from the ceiling and falling to hit them in the middle of the forehead.

Can we learn from our mistakes when facing melanocytic lesions? Almost impossible! Only in the rare event that a patient dies due to metastatic spreading of the disease, one can be sure to have missed a melanoma. Meanwhile, it is quite different when we talk about cutaneous lymphomas and inflammatory conditions. Patients do not disappear, they will come back to you. It is a slow diagnostic process. We can learn from our diagnostic errors by comparing the previous histologic slide with a second biopsy, and make a new diagnosis that better describes the clinical progress. A surgeon's performance is easily evaluated because results occur shortly after the surgery. With melanocytic lesions, it is not possible. "One shot only" a diagnosis of Spitz is made and ... then the patient disappears. The studies regarding melanoma vs nevus are based on very rare and complex cases with a thickness larger than $0.75 \mathrm{~mm}$. They have relatively short follow-ups, rarely exceed fifty cases, and the histological slides have been evaluated only by the pathologists who wrote the report. Others can only acknowledge the report, but they cannot deeply internalize a personal diagnosis, be it right or wrong. So we cannot know who among Dr. Riccardo from Rome, Dr. Erica from Pasadena, Dr. Federico from Genoa or Dr. Arianne from Hannover have under- diagnosed melanoma cases or overdiagnosed melanocytic nevi every so often.

A statistical theorem called "Single shoot theory" was developed by Russian mathematicians to train Olympic rifle shooting athletes, a discipline in which our friends from the east have always been masters. These Olympic competitions are held on sixty shots and the theorem relies on convincing the athletes to be constantly super-focused as if it was not a single competition of sixty shots, but sixty one-shot competitions. What is the meaning of all this? Quite simply: an average shooter at the fifteenth shot could be faring on a slightly better score than an Olympic athlete who momentarily lost focus. However, at the end of the competition, the champion would still finish up "miles" ahead of an average shooter. Likewise, in a hypothetical competition with Bernie Ackerman, if we had conflicting opinions on a single complex case, I could be the one that's ahead. But stretch our competition on a year's work, thousands of difficult cases examined, and I would be embarrassingly behind in the number of correctly diagnosed cases. Think about this when you send a complex case in consultation to a more expert colleague and his diagnosis wasn't convincing. That case could be your 15 th shot, so remember to learn from the mistakes of the more experienced. "a mistake is an error, but an error is not always a consequence of carelessness or indifference. On the contrary, mistakes can be made when great care has been exercised." Bernie Ackerman 